\title{
The present moment awareness of Mindfulness in Dermatology
}

Mindfulness originates from Buddhism. During the 1970s Professor Kabat-Zinn founded mindfulness based stress reduction (MBSR) ${ }^{1}$; a secular, 8-week, group based intervention aimed to reduce stress. Over the past 35 years several mindfulness programmes have emerged targeting physical and mental health. One of the most established programmes is mindfulness based cognitive therapy (MBCT) ${ }^{2}$. This differs from MBSR because it is delivered within a cognitive framework of understanding. However, MBCT is distinct from cognitive behavioural therapy (CBT), which identifies and challenges maladaptive thoughts/beliefs. All mindfulness based approaches offer an alternative standpoint from which to watch our thoughts, behaviours and emotions but they do not deliver techniques to change these as CBT does.

The key tenets of mindfulness are developing present moment attention and accepting what is found. It instigates a nuanced shift in perspective and allows people a chance to respond to events rather than living in a perpetual state of automatic reaction. When considering the lived experience of many people with dermatological conditions the process of acknowledging automatic reactions (assuming a person is disgusted by them or itching behaviours) and developing an acceptance of physical and mental pain seem to fit the patient profile well. Evidence supports mindfulness for improving mental health ${ }^{3}$ and certain biological markers including inflammatory responses ${ }^{4}$.

Due to the dominant stress-psoriasis rhetoric mindfulness was explored with this condition over 18 years ago. Professor Kabat-Zinn reported a small study $(n=37)$ which found that the rate of psoriatic skin clearance during phototherapy was increased with the adjunct of mindfulness therapy ${ }^{5}$. A pilot study $(n=29)$ to explore the efficacy and acceptability of MBCT for patients with psoriasis, reported a significant improvement in self-assessed clinical severity and quality of life ${ }^{6}$ and the patients found this to be an acceptable and useful adjunct to their normal therapy ${ }^{7}$.

In the current issue of the British Journal of Dermatology, an insightful article by Montgomery et al ${ }^{8}$ examined the relationship between naturally occurring levels of mindfulness and psychosocial distress in dermatology patients. The article reports a robust association: levels of mindfulness, measured with the Five Facet Mindfulness Questionnaire ${ }^{9}$, were significantly, negatively associated with levels of psychosocial stress (Hospital Anxiety Depression Scale ${ }^{10}$ ). Regression analyses were run controlling for subjective skin severity. Mindfulness explained variance in shame, social anxiety, anxiety, depression and quality of life. As a cross sectional analysis we cannot infer causality, however, it raises a question of whether mindfulness could protect patients with dermatological conditions from developing psychosocial distress.

The paradigm of separate biomedical and psychosocial care is shifting into a biopsychosocial model. Dermatological conditions generate physical and psychosocial symptoms. Stress reduction interventions, such as mindfulness, could produce beneficial physical and psychological consequences. The preliminary work has been done and we need to develop well designed large trials examining the effectiveness of mindfulness adjunct therapies in dermatological conditions where the patient profile needs match the theoretical formulation of mindfulness.

\section{References}

1 Kabat-Zinn, J. (1982) An outpatient program in behavioural medicine for chronic pain patients based on the practice of mindful meditation: Theoretical considerations and preliminary results. General Hospital Psychiatry 4: 33-47

2 Segal Z. V., Williams J. M. G., \& Teasdale J. D. (2002). Mindfulness-based cognitive therapy for depression: A new approach to preventing relapse. New York: Guilford

3 Bohlmeijer, Ernst, et al. "The effects of mindfulness-based stress reduction therapy on mental health of adults with a chronic medical disease: a meta-analysis." Journal of psychosomatic research 68.6 (2010): 539-544.

4 Shennan, Christina, Sheila Payne, and Deborah Fenlon. "What is the evidence for the use of mindfulness- based interventions in cancer care? A review." Psycho- Oncology 20.7 (2011): 681697. 
5 Kabat-Zinn, Jon, et al. "Influence of a mindfulness meditation-based stress reduction intervention on rates of skin clearing in patients with moderate to severe psoriasis undergoing photo therapy (UVB) and photochemotherapy (PUVA)." Psychosomatic medicine 60.5 (1998): 625-632.

6 Fordham, B., C. E. M. Griffiths, and C. Bundy. "A pilot study examining mindfulness-based cognitive therapy in psoriasis." Psychology, health \& medicine 20.1 (2015): 121-127.

7 Fordham, B. A., et al. "The acceptability and usefulness of mindfulness- based cognitive therapy for people living with psoriasis: a qualitative study." British Journal of Dermatology 172.3 (2015): 823-825.

8 Montgomery, K., Norman, P., Messenger, A.G. and Thompson, A.R. (2016) The importance of mindfulness in psychosocial distress and quality of life in dermatology patients. British Journal of Dermatology (In publication)

9 Christopher, M S., et al. "Exploring the psychometric properties of the five facet mindfulness questionnaire." Mindfulness 3.2 (2012): 124-131.

10 Zigmond, AS., and Snaith, RP. "The hospital anxiety and depression scale." Acta psychiatrica scandinavica 67.6 (1983): 361-370. 